\title{
Occurrences of Metalo- $\beta$-lactamase Producing Pseudomonas aeruginosa among Clinical Samples in Kwara state, Nigeria
}

\author{
${ }^{* 1}$ ADEKUNLE, OC; ${ }^{2}$ MUSTAPHA, A; ${ }^{1}$ ODEWALE, G; ${ }^{1}$ OJEDELE, RO \\ ${ }^{*}$ Department of Medical Microbiology and Parasitology, Ladoke Akintola University of Technology, Osogbo, Nigeria \\ ${ }^{2}$ Department of Microbiology, Kwara State University, Ilorin, Kwara State, Nigeria. \\ *Corresponding Author Email: ocadekunle@lautech.edu.ng, toyintoro@yahoo.com; Tel.; +2348066586726
}

\begin{abstract}
Pseudomonas aeruginosa is a frequent nosocomial pathogen that causes severe disease in many clinical and community settings. The objective of this paper was to isolate Pseudomonas aeruginosa from clinical samples and to investigate the occurrence of metallo $\beta$ - lactamase enzyme production by collecting 145 males and 90 females' human clinical specimens from five selected health institutions within Kwara state, Nigeria. The samples were cultured immediately using standard microbiological procedures. Multiple drug resistance patterns of the bacteria to different antibiotics were determined using the Bauer Kirby disc diffusion technique. Metallo $-\beta$ lactamase production was determined using E - test strip. Data were subjected to descriptive statistics using Statistical Package for Social Sciences (SPSS) software. A total of 145 isolates were identified as Pseudomonas aeruginosa from the clinical samples.Thirty were positive for metallo $\beta$ lactamase production; $11(8 \%)$ males and $19(13 \%)$ females. Absolute resistance to ceftazidime $(100 \%)$, gentamicin $(100 \%)$, ceftriaxone $(100 \%)$ were observed while low resistance to ciprofloxacin (12.4\%), piperacillin $(6.9 \%)$ and imipenem $(6.9 \%)$. All isolates were sensitive to colistin. This study had demonstrated that there is a high occurrence of metallo $\beta$ lactamase enzyme producing and antibiotic-resistant strains of $P$. aeruginosa in clinical specimens from the studied area. Necessary measures must be implemented to stop the problems of this antibiotic resistance.
\end{abstract}

\section{DOI:https://dx.doi.org/10.4314/jasem.v25i10.6}

Copyright: Copyright (C) 2021 Adekunle et al. This is an open access article distributed under the Creative Commons Attribution License (CCL), which permits unrestricted use, distribution, and reproduction in any medium, provided the original work is properly cited.

Dates: Received: 22 August 2021; Revised: 17 September 2021; Accepted: 06 October 2021

Keywords: Metalo- $\beta$-lactamase enzyme, P. aeruginosa, clinical samples, antibiotic-resistant genes.

Pseudomonas aeruginosa is widespread in natural environments and considered an opportunistic secondary pathogen for humans that is capable of causing a major nosocomial infections and broad spectrum of infections such as urinary tract, burn, respiratory tract, meningitis, chronic otitis media and otitis externa, pseudomonal endocarditis, septicaemia, etc. (Singh et al., 2006; Yang et al., 2011). It is also implicated in $16 \%$ of nosocomial pneumonia, $12 \%$ in urinary tract infections, $8 \%$ in surgical infections, and $10 \%$ in bloodstream infections. Otherwise, there is increasing evidence implication of the species in foodborne infections (Vitkauskienè et al., 2010). Pseudomonas aeruginosa is not only but one of the main causes of healthcare-associated infections mostly common among hospitalized patients (Kyaw et al., 2015). Healthcare-associated infections predominantly lead to pneumonia, urinary tract infections as well as, skin and soft-tissue infections. Occasionally, $P$. aeruginosa can colonise human body sites, with a preference for moist areas, such as the perineum, axilla, ear, nasal mucosa and throat, stool as well as subungual areas of the hands (Hilmar et al., 2018). The prevalence of colonisation in healthy individuals is usually low, higher colonisation rates can be encountered following hospitalisation, especially among patients treated with broad-spectrum antibiotics. A multidrug resistant (MDR) $P$. aeruginosa phenotype is defined as a bacterium which is resistant to three or more anti-pseudomonal antimicrobial classes; carbapenems, fluoroquinolones, penicillins / cephalosporins and aminoglycosides (Magiorakos et al., 2012). The mechanism of resistance in MDR $P$. aeruginosa is possibly through the production of several enzymes that inactivate betalactams and carbapenems such as extended spectrum beta lactamases (ESBLs) and metallo- $\beta$-lactamases (MBLs) (Vahdani et al., 2012). Therefore, this study was aimed at isolating Pseudomonas aeruginosa from clinical samples and investigating the occurrence of metallo $\beta$ - lactamase enzyme among them.

\section{MATERIALS AND METHODS}

One hundred and forty five clinical specimens of Pseudomonas aeruginosa isolates were isolated from urine, wound, sputum, blood and indwelling medical devices. Specimens were collected from primary, secondary, tertiary hospitals, and private diagnostic centres across Kwara state, Nigeria

Ethical consideration: Ethical approval was obtained from the Ethical Review Committee (ERC) of Kwara State Ministry of Health, Ilorin.

Clinical samples were collected from different hospital wards of University of Ilorin Teaching Hospital, the samples include: urine, sputum, catheter tip, cornea scraping, blood, wound swab and ear swab. They were 
processed, cultured and the bacteria identified at the Medical Microbiology and Parasitology laboratory unit of University of Ilorin Teaching Hospital (UITH) Ilorin.

Innoculation and Incubation: The clinical samples were collected into a transport media (Nutrient broth) and it was further transported into the laboratory. The culture was incubated overnight at $42{ }^{\circ} \mathrm{C}$ for 24 hours. The overnight broth culture was futher sub-cultured on MacConkey agar and Blood Agar. Incubation of the cultured plates was done under aerobic conditions. The bacterial growth was observed for colonies morphologically resembling Pseudomonas aeruginosa on MacConkey agar in aerobic conditions. Colonies morphologically resembling $P$. aeruginosa were subjected to further characterization.

Confirmation of Pseudomonas aeruginosa strains: Production of pigments such as pyocyanin was tested by growing the isolates on cetrimide agar for confirmation results obtained were compared with specifications in Bergey's Manual of Systematic Bacteriology.

Antimicrobial susceptibility testing: Antibiotic susceptibility testing was performed using modified Kirby-Bauer disc diffusion method following guidelines of Clinical and Laboratory Standards Institute (CLSI, 2015).

Metalo- $\beta$-lactamase enzyme detection: The inoculum of Pseudomonas aeruginosa was prepared (CLSI, 2015). Each isolates of $P$. aeruginosa was inoculated on a separate Mueller Hinton plate. MBL E-test strips containing concentration gradient $(1-64 \mu \mathrm{g} / \mathrm{ml})$ of imipenem (IP) on one end of the strip and imipenem overlaid with a constant concentration of EDTA (IPI) on the other end of the strip were placed onto the plate and incubated at $42^{\circ} \mathrm{C}$ aerobically for $18-24$ hours as shown in plates $3.7 \mathrm{a}$ and $3.7 \mathrm{~b}$. Negative control was set along with the test using $P$. aeruginosa ATCC 27853. A reduction in the Imipenem minimum inhibitory concentration (MIC) in the presence of EDTA of greater than or equal to eight-fold (IP/IPI $\geq$ 8) indicated MBL positivity.

\section{RESULTS AND DISCUSSION}

Out of the $145 P$. aeruginosa isolates examined. Table 1 shows the distribution of $P$. aeruginosa according to the sources of infection which include urine, sputum, cornea scrapping, wound swab, ear swab, catheter tip and vaginal swab. $P$. aeruginosa isolates $(32.5 \%)$ were mostly isolated from ear infection, which was followed by wound infection. The lowest percentage of isolates was found in catheter tip. $30(21 \%)$ were MBL producing $P$. aeruginosa while $115(79 \%)$ were negative for MBL production. Of the $30 \mathrm{MBL}$ positive isolates the male population account for about $11(8$ $\%$ ) while the female population account for $19(13 \%)$ while 115 isolates were found to be negative for MBL production in which the female population accounts for $63(43 \%)$ while $52(36 \%)$ was found in the male population this is shown in Table 2. During the study period, MBL positive isolates were found to be resistant to ceftriaxone and ceftazidime $(100 \%)$ while a relative decrease in the susceptibility pattern of the MBL negative isolates to ceftriaxone and ceftazidime with 55 isolates $(37.9 \%)$ susceptible and 90 isolates $(62.1 \%)$ respectively as presented in Table 3 . Of the 30 MBL positive isolates, $18(12.4 \%), 5(3.4 \%)$ were susceptible to ciprofloxacin and gentamicin? while 12 (8.3\%), $15(10.3 \%)$ were resistant for ciprofloxacin and gentamicin, respectively. In addition, out of the 115 MBL negative isolates, 67 (46.2\%) and 100 (69.0 $\%)$ were susceptible to ciprofloxacin and gentamicin respectively.

Table 1: Distribution of $P$. aeruginosa according to the sources of

\begin{tabular}{ll}
\multicolumn{2}{c}{ infection } \\
\hline $\begin{array}{l}\text { Sources of } \\
\text { Pseudomonas } \\
\text { aeruginosa }\end{array}$ & Frequency (\%) \\
\hline Urine & $10(14.5)$ \\
Sputum & $15(21.8)$ \\
Cornea Scrapping & $8(11.6)$ \\
Wound Swab & $36(52.2)$ \\
Ear swab & $40(58)$ \\
Catheter tip & $5(7.25)$ \\
Vaginal Swab & $32(46.4)$ \\
Total & 145 \\
\hline
\end{tabular}

Table 2: The gender distribution of patients infected with $P$.

\begin{tabular}{lll}
\multicolumn{3}{c}{ aeruginosa. } \\
\hline & MBL & $\begin{array}{l}\text { Non MBL } \\
\text { Producer (\%) }\end{array}$ \\
\hline Male & Producers (\%) & $52(35.9)$ \\
Female & $19(13)$ & $63(47.6)$ \\
Total & $30(21)$ & $115(79)$ \\
\hline
\end{tabular}

Plate 1 displays the photograph of MBL positive $P$. aeruginosa showing a phantom zone which is characteristic of MBL producers while plate 2 displays the photograph of $\mathrm{MBL}$ positive $P$. aeruginosa showing IP/IPI $\geq 8$

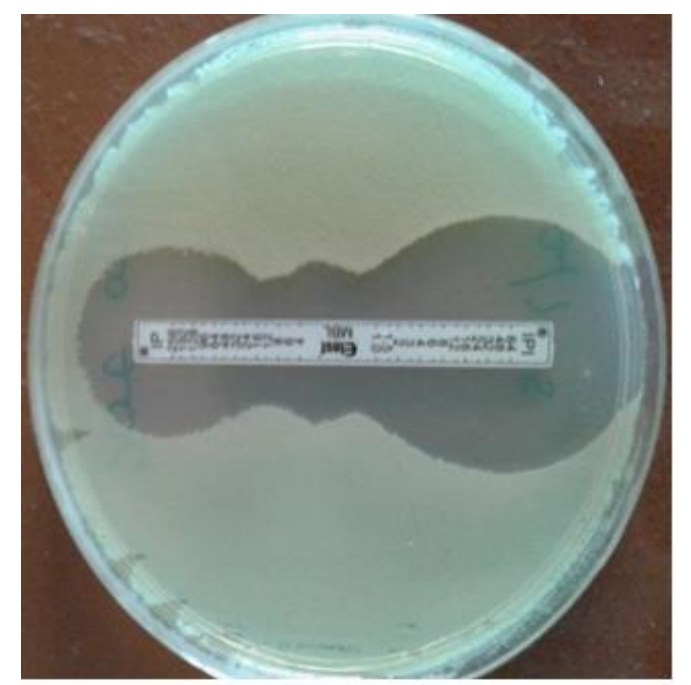

Plate 1: Photograph of MBL positive $P$. aeruginosa showing a phantom zone which is characteristic MBL producers. 
Table 3: Frequency of Antibiotics susceptibility pattern of MBL and Non MBL P. aeruginosa.

\begin{tabular}{|c|c|c|c|c|c|}
\hline Antibiotics Used & $\begin{array}{l}\text { Total number } \\
\text { of Isolates } \\
\mathrm{N}=145\end{array}$ & $\begin{array}{l}\text { MBL } \\
\text { Producers } \\
\mathrm{N}=\mathbf{3 0}(\%)\end{array}$ & $\begin{array}{l}\text { Non MBL } \\
\text { Producers } \\
115(\%)\end{array}$ & $\mathbf{X}^{2}$ & p-value \\
\hline \multirow[t]{3}{*}{ Ceftazidime } & $\mathrm{S}$ & $0(0)$ & $90(62.1)$ & 66.552 & 0.000 \\
\hline & I & $0(0)$ & $10(6.9)$ & & \\
\hline & $\mathrm{R}$ & $30(100)$ & $15(10.3)$ & & \\
\hline \multirow[t]{3}{*}{ Gentamicin } & $\mathrm{S}$ & $0(0)$ & $100(69.0)$ & 288.448 & 0.000 \\
\hline & I & $0(0)$ & $5(3.4)$ & & \\
\hline & $\mathrm{R}$ & $30(100)$ & $10(6.9)$ & & \\
\hline \multirow[t]{3}{*}{ Ciprofloxacin } & $\mathrm{S}$ & $18(12.4)$ & $67(46.2)$ & 68.138 & 0.000 \\
\hline & I & $12(8.3)$ & $18(12.4)$ & & \\
\hline & $\mathrm{R}$ & $0(0)$ & $30(20.7)$ & & \\
\hline \multirow[t]{3}{*}{ Ceftriaxone } & $\mathrm{S}$ & $0(0)$ & $55(37.9)$ & 38.621 & 0.000 \\
\hline & I & $0(0)$ & $15(10.3)$ & & \\
\hline & $\mathrm{R}$ & $30(100)$ & $45(31)$ & & \\
\hline \multirow[t]{3}{*}{ Piperacillin/tazobactam } & $\mathrm{S}$ & $10(6.9)$ & $100(69.0)$ & 251.207 & 0.000 \\
\hline & I & $5(3.4)$ & $5(3.4)$ & & \\
\hline & $\mathrm{R}$ & $15(10.3)$ & $10(6.9)$ & & \\
\hline \multirow[t]{3}{*}{ Imipenem } & $\mathrm{S}$ & $10(6.9)$ & $95(65.5)$ & 251.207 & 0.000 \\
\hline & I & $5(3.4)$ & $10(6.9)$ & & \\
\hline & $\mathrm{R}$ & $15(10.3)$ & $10(6.9)$ & & \\
\hline \multirow[t]{3}{*}{ Colistin } & $\mathrm{S}$ & $25(17.2)$ & $112(77.2)$ & 219.221 & 0.000 \\
\hline & I & $0(0.0)$ & $0(0.0)$ & & \\
\hline & $\mathrm{R}$ & $5(3.4)$ & $3(2.1)$ & & \\
\hline
\end{tabular}

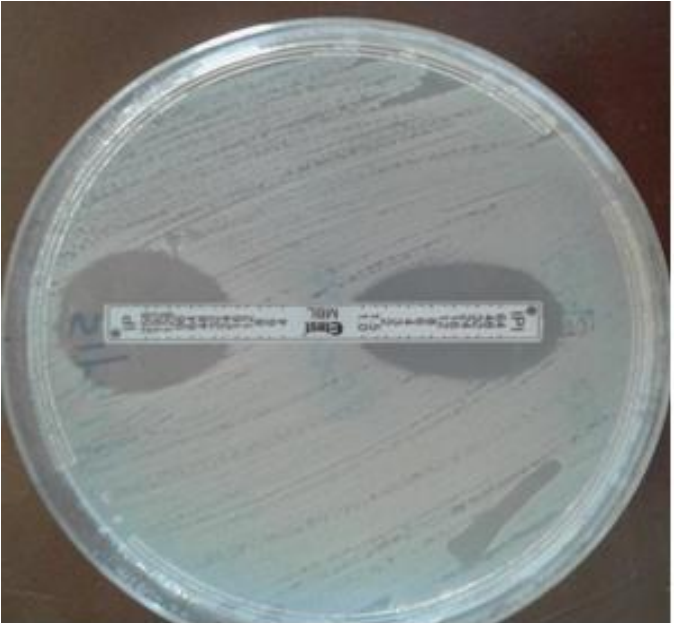

Plate 2: Photograph of MBL positive $P$. aeruginosa showing $\mathrm{IP} / \mathrm{IPI}>8$

Pseudomonas aeruginosa is commonly implicated as a cause of health care acquired infections with high mortality rates (Lambert et al., 2011). The high rate of microbial resistance to the cephalosporins as observed in this study is a cause for concern as because many clinicians fall back on them for the treatment of Gramnegative pathogens in the face of multi-drug resistance. The high incidence of the isolates resistant to the cephalosporins and quinolones may be attributed to cross-resistance to the class occasioned by high nonprescription use of ciprofloxacin in the area as well as possible drug faking. The results of the antibiotic resistance profile of the isolates to beta-lactam antibiotics showed increased beta-lactamase enzyme production among the isolates. Many of the MBL producing isolates were resistant to ceftriaxone $(100$ $\%)$, ceftazidime and ciprofloxacin (100\%) respectively, Anupurba et al. (2006) reported the isolation of cephalosporin resistant $P$. aeruginosa among in-patients and outpatient clinics, made a similar observation. In this study the prevalence of MBL producing P.aeruginosa was found to be $21 \%$. This result was lower than $36.07 \%$ reported by Sasirekha et al., 2010 and higher than the prevalence reported in Lagos (Aibinu et al., 2007), and in Belgium (Berges et al., 2007) where the prevalence was $4.1 \%$ and $4.4 \%$, respectively. It is also higher than the prevalence of $10.0 \%$ and $14.0 \%$ obtained in Enugu, south east, Nigeria (Ejikwegu et al., 2014) and India (Hemalatha et al., 2005), respectively.

The prevalence of MBL producing $P$. aeruginosa in male to female in this study is $7.59 \%$ as compare to $13.1 \%$. This is in line with the study by Mojtaba et al. (2014). Females were more affected than males (53\% to $47 \%$ ). This is in contrast with the study carried out by Sandhya and Swathi, (2015) reported 7:1. Some studies shows that more males are infected with $P$. aeruginosa than female (Sandhya and Swathi, 2015)

The highest number of susceptibility of $P$. aeruginosa isolates was recorded for colistin $(77.2 \%)$ followed by imipenem $(65.5 \%)$. This result for colistin contradicts studies from Nwankwo and Shaibu (2010) who reported $78.9 \%$ susceptibility of $P$. aeruginosa but corroborate result of Aibinu et al. (2007) and Olayinka et al. (2009) who reported $95.6 \%$ and $94.6 \%$ respectively. They explained that the duration of the hospital stay was directly proportional to a higher prevalence of the infection. Aminoglycosides (streptomycin and gentamicin), on the average, had less activity and showed more microbial resistance profile compared to $\beta$-lactam drugs and carbapenem. The reason for this high resistance to the aminoglycosides could be as a result of indiscriminate use of gentamicin in the area. The drug though a prescription only medicine is purchased as over-thecounter in the open markets in the community and is commonly used by unqualified personnel in the 
treatment of "infections". Several studies show that gentamicin and streptomycin are effective against Pseudomonas species and E. coli, but if misused, the organisms may also develop resistance to them. This research findings is similar to that of Sasirekha et al. (2010) where they recorded $82.1 \%$ susceptibility of the isolates to gentamicin and $41.8 \%$ to streptomycin probably although they worked on clinical isolates generally and not on ESBL producers alone.

The high resistance profile of the isolates in this study is a reflection of the high incidence of MBL and ESBL isolates observed. $P$. aeruginosa, much like Mycobacterium tuberculosis is intrinsically resistance to several antimicrobial classes and therefore poses considerable limitation in the range of antibiotic options for treating infections caused by them. These increase further the risk for emergence of resistant strains. Bacteria usually encode multiple antibiotic resistance genes.

MBL producers were found more in females than males, this may be due to the fact that women are relatively weaker and more sensitive. The results of susceptibility pattern of $P$. aeruginosa isolates to antimicrobial agents in this study showed that the 145 $P$. aeruginosa strains tested against seven antimicrobial agents; shows that all the MBL strains of the isolates were resistant ceftazidime, and gentamicin recording $100 \%$. Piperacillin and imipenem have the same sensitivity pattern for MBL while Colistin has the highest percentage of sensitivity with few isolates resistant to the drugs this is because it is an amphiphatic drug. That is why it is always used as a last resort to multi drug resistance strains.

The result of this study showed that there is an increase in resistance to the quinolones by strains of $P$. aeruginosa. In previous studies by Oduyebo et al. (1997) and Kesah et al.(1999) in this environment, $P$. aeruginosa strains were found to be highly susceptible to the quinolones (96\%); but from this study, our data shows increase in resistance to this family of antibacterial agents by the current clinical strains of $P$. aeruginosa. Ofloxacin was observed to have the highest activity (41\%) and was closely followed by ciprofloxacin (39\%). This result implies that quinolones alone, cannot be depended upon as an antipseudomonal antimicrobial in this environment. They will have to be used in combination or replaced with another antimicrobial preferably. The activities of carbapenems, ceftazidime, piperacillin/tazobactam and amikacin against $P$. aeruginosa strains is still high and they can be considered as therapeutic options available for $P$. aeruginosa infection treatment in this region.

Conclusion: Metallolo- $\beta$-lactamase producing $P$. aeruginosa strains were found in Kwara State which is the North central Nigeria and result of this work shows the presence of this enzyme in the pathogen, $P$. aeruginosa in environment which may also confer resistance on the organism.

\section{REFERENCES}

Aibinu, I; Osundiya, OO; Oladele, RO; Oduyebo, OO (2013).Multiple Antibiotic Resistance (MAR) Indices of Peudomonas and Klebsiella species Isolates in Lagos University Teaching Hospital. Afr. J. Clin. Exp. Microbiol. 14, 164-168.

Anuradha, B; Uzma, A; Sheeba Paveena, M (2014).Evaluation of Antimicrobial Susceptibility Pattern of Pseudomonas aeruginosa With Special Reference to MBL Production in Tertiary Care Hospital. Global Journal of Medical Res. 14 (7),

Bergès, L; Rodriguez-Villalobos, H; Deplano, A; Struelens, MJ (2007). Prospective evaluation of imipenem/EDTA combined disc and Etest for detection of metallo- $\beta$-lactamase-producing Pseudomonas aeruginosa. J. Antimicrob. Chemother. 59(4), 812-813.

CLSI (2016). Performance Standards for Antimicrobial Susceptibility Testing, $26^{\text {th }}$ ed. CLSI supplement M100S, Wayne, PA: Clinical and Laboratory Standards Institute.

Edelstein, MV; Skleenova, EN; Shevchenko, OV; D'souza, JW; Tapalski, DV; Azizov, IS; Sukhorukova, MV; Pavlukov, RA; Kozlov, RS; Toleman, MA; Walsh, TR (2013). Spread of extensively resistant VIM-2-positive ST235 Pseudomonas aeruginosa in Belarus, Kazakhstan, and Russia: a longitudinal epidemiological and clinical study. Lancet Infect. Dis. 13(10), 867876 .

Lavoie, EG; Wangdi, T; Kazmierczak, BI (2011). Innate immune responses to Pseudomonas aeruginosa infection. Microbes Infect. 13(14-15), 1133-1145.

Nwanneka, T; Odugbemi, T (2007). Occurence of ESBL and MBL in clinical isolates of Pseudomonas aeruginosa from Lagos, Nigeria. $J$. Am. Sci.3 (4), 81-85.

Oduyebo, O; Ogunsola, FT; Odugbemi, T (1997). Prevalence of Multi - Resistant Strains of Pseudomonas aeruginosa Isolated At The Lagos University Teaching Hospital Laboratory. Nigeria Journal of Hospital Med. 7(4), 1-4.

Poorabbas, B; Mardaneh, J; Rezaei, Z; Kalani, M; Pouladfar, G; Alami, MH; Soltani, J; ShamsiZadeh, A; Abdoli-Oskooi, S; Saffar, MJ; Alborzi, 
A (2015). Nosocomial Infections: Multicenter surveillance of antimicrobial resistance profile of Staphylococcus aureus and Gram negative rods isolated from blood and other sterile body fluids in Iran. Iran. J. Microbiol. 7(3), 127-135.

Sasirekha, B; Manasa, R; Ramya, P; Sneha, R (2010). Frequency and Antimicrobial Sensitivity Pattern of Extended Spectrum Beta- Lactamases Producing E.coli and Klebsiella pneumonia Isolated in a Tertiary Hospital. Al Ameen J. Med. Sci. 3(4), 265-271.
Strateva, T; Yordanov, D (2009). Pseudomonas aeruginosa - a phenomenon of bacterial resistance. J. Med. Microbiol. 58(9), 1133-1148.

Tiwari, N; Rajdev, S; Mullan, S (2017). Resistance Trends among 1t;igt; Pseudomonas aeruginosa 1t;/igt; Isolates in a Tertiary Care Centre in South Gujarat. Adv. Microbiol. 7(3), 188-194.

Yang, Y, Cochran, DA, Gargano, MD, King, I, Samhat, NK, Burger, BP, Sabourin, KR, Hou, Y, Awata, J, Parry, DA, Marshall, WF, Witman, GB, Lu, X (2011). Regulation of flagellar motility by the conserved flagellar protein CG34110/Ccdc135/FAP50. Mol. Biol. Cell 22(7): 976--987. 